\title{
Darstellung und Analyse einiger Nucleinsäuren.
}

(Vierte Mitteilung.)

Von

P. A. Levene.

(Aus der physiologisch-chemischen Abteilung des pathologischen Instituts der New-Yorker Staatskrankenhäuser.)

(Der Redaktion zugegangen am 19. Mai 1903.)

Bei der Spaltung der Pankreasnucleinsäure mit 25\%iger Schwefelsäure ist es bis jetzt gelungen, 2 Pyrimidinbasen zu gewinnen, nämlich das Thymin und das Cytosin. Letztere Base wurde nur als Picrat untersucht. Seit dem ist es aber Kossel und Steudel gelungen, aus Thymusnucleinsäure und aus Spermonucleinsäure auch das Uracil darzustellen. Auch bei der Spaltung der Milznucleinsäure konnte dieser Körper aufgefunden werden. Es war von Wichtigkeit, die Nucleinsäure der Pankreasdrüse in derselben Richtung zu untersuchen. Dies schien um so mehr wünschenswert, als das Verfahren zur Darstellung und Trennung dieser Substanzen noch vereinfacht wurde.

Die Analyse der Pankreasnucleinsäure wurde nun auf folgende Weise ausgeführt. Die Spaltung ist wie bisher mit $25 \%$ iger Schwefelsäure im Autoklaven bei $175^{\circ} \mathrm{C}$. bewirkt worden. Die Schwefel- und Phosphorsäure werden mit heißem, gesättigtem Barytwasser entfernt, und das Filtrat und Waschwasser bei vermindertem Druck eingeengt, wobei man für sorgfältiges Auswaschen der Barytniederschläge Sorge tragen muß. Bei 24 stündigem Stehen scheidet sich ein Niederschlag aus, der hauptsächlich aus Thymin besteht. Um das reine Thymin zu erhalten, wird der Niederschlag aus verdünnter Schwefelsäure umkrystallisiert. Das erhaltene Präparat hatte 
( das typische Aussehen des Thymins und ergab bei der Analyse c die folgenden Zahlen:

$0,1500 \mathrm{~g}$ der Substanz gaben 28,5 Stickstoff bei $\mathrm{p}=768$ und $\mathrm{t}^{\circ} 19^{\circ} \mathrm{C}$.

Berechnet für $\mathrm{C}_{5} \mathrm{H}_{6} \mathrm{~N}_{2} \mathrm{O}_{2}$ :

N 22,22

Gefunden:

22,49

Das Filtrat wurde mit Schwefelsäure vom Baryt befreit, c dann mit einer gesättigten Pikrinsäurelösung versetzt und über INacht stehen gelassen. Es bildete sich dabei ein Niederschlag, cder hauptsächlich aus pikrinsaurem Cytosin bestand. Er wurde $\mathrm{i}$ in heißem Wasser gelöst, vom unlöslichen Reste abfiltriert, tund das Filtrat heiß mit Schwefelsäure und Toluol zersetzt. $\downarrow$ Als die Pikrinsäure fast vollständig mit Äther entfernt war, vwurde die Lösung in Vacuo konzentriert, der Überschuß der ¿Schwefelsäure mit Barytwasser entfernt, das Filtrat wieder ceingeengt, und die Lösung der Krystallisation überlassen. Es rbildete sich dabei ein Niederschlag, der aus langen prismattischen Nadeln bestand. Beim Eindunsten der Mutterlauge wwurde ein zweiter Niederschlag erhalten.

Die zwei Niederschläge repräsentieren zwei Sulfate des (Cytosins, das minder lösliche ist das basische Salz von der $\mathrm{Zu}$ ssammensetzung $\left(\mathrm{C}_{4} \mathrm{H}_{5} \mathrm{~N}_{3} \mathrm{O}\right)_{4} \mathrm{H}_{2} \mathrm{SO}_{4}+2 \mathrm{H}_{2} \mathrm{O}$, und das andere ist cdas schon früher beschriebene Salz $\left(\mathrm{C}_{4} \mathrm{H}_{5} \mathrm{~N}_{8} \mathrm{O}\right)_{2} \mathrm{H}_{2} \mathrm{SO}_{4}$.

Die Analyse des basischen Salzes ergab die folgenden 'Zahlen :

$0,1313 \mathrm{~g}$ der Substanz gaben $0,1593 \mathrm{~g} \mathrm{CO}_{\mathrm{z}}$ und 0,0592 $\mathrm{H}_{2} \mathrm{O}$.

$0,1418 \mathrm{~g}$ der Substanz gaben $35,0 \mathrm{ccm}$ Stickstoff bei $\mathrm{p}=767$ und $\mathrm{t}^{\mathrm{0}}=20^{\circ} \mathrm{C}$.

$0,1370 \mathrm{~g}$ der Substanz gaben $0,0585 \mathrm{~g} \mathrm{BaSO}_{4}$.

Berechnet für $\left(\mathrm{C}_{4} \mathrm{H}_{5} \mathrm{~N}_{3} \mathrm{O}\right)_{4} \mathrm{H}_{2} \mathrm{SO}_{4} \cdot 2 \mathrm{H}_{z} \mathrm{O}$ :

Gefunden:

$\begin{array}{rrr}\mathrm{C} & 33,22 \% & 33,07 \% \\ \mathrm{H} & 4,49 \% & 4,15 \% \\ \mathrm{~N} & 29,06 \% & 29,12 \% \\ \mathrm{~S} & 5,53 \% & 5,88 \%\end{array}$

Ein Teil des Sulfats wurde in das Chloroplatinat übergreführt.

$0,1390 \mathrm{~g}$ der Substanz gaben beim Glühen $0,0425 \mathrm{~g} \mathrm{Pt}$.

Berechnet für $2\left(\mathrm{C}_{4} \mathrm{H}_{5} \mathrm{~N}_{3} \mathrm{O}\right) \mathrm{PtCl}_{4} \cdot 2 \mathrm{HGl}$ :

Gefunden:

Pt $30,84 \%$ $30,56 \%$

Es lag also ohne Zweifel Cytosin vor. 
Aus der Mutterlauge des Cytosinpicrates wurde die Picrinsäure mit Schwefelsäure und Äther, und die Schwefelsäure mit Baryt entfernt. Das Filtrat gab dann mit Silber und Baryt einen Niederschlag, der in gewöhnlicher Weise vom Silber be- freit wurde. Das Filtrat wurde, wie in der vorigen Mitteilung angegeben, behandelt, und es entstand dabei ein Niederschlag, der das Aussehen des Uracils besaß, und der bei der Analyse die folgenden Zahlen gab:

$0,1249 \mathrm{~g}$ der Substanz gaben $26,75 \mathrm{ccm}$ Stickstoff bei $\mathrm{p}=767$ und $t^{0}=21^{\circ} \mathrm{C}$.

$$
\begin{array}{cc}
\text { Berechnet für } \mathrm{C}_{4} \mathrm{H}_{4} \mathrm{~N}_{2} \mathrm{O}_{2}: & \text { Gefunden : } \\
25,05 \% & 25,20 \%
\end{array}
$$

Zur Spaltung wurden etwa $150 \mathrm{~g}$ der Nucleinsäure verbraucht und dabei entstanden:

$$
\begin{array}{ll}
\text { Thymin } & 5,0 \mathrm{~g} \\
\text { Cytosinpicrat } & 6,0 \\
\text { Uracil } & 1,0
\end{array}
$$

Damit ist der Beweis erbracht, daß die Pankreasnucleinsäure aus denselben Komponenten der Pyrimidin- und Purinreihe besteht, wie die andere gegenwärtig analysierte Nucleinsäure tierischer Herkunft. Man darf aber nicht daraus mit Sicherheit folgern, daß alle Nucleinsäuren identisch sind.

\section{Pyrimidenbasen der Hefenucleinsäure.}

Was die Hefenucleinsäure betrifft, liegen nun die folgenden Angaben vor:

Kossel und Neumann haben aus ihr eine kleine Menge einer Substanz isoliert, welche die Eigenschaften des Thymins besaß. Für eine eingehende Analyse reichte aber die Substanz nicht aus. Kossel veranlaßte dann Ascoli, den Körper weiter zu untersuchen. Es gelang Ascoli, dabei das Uracil zu entdecken. Auf Grund der Zahlen der Analyse nahm er an, daß die Base mit Thymin verunreinigt war.

Auch bei der Spaltung der Nucleinsäure des Weizenembryos vermißten Osborne und Harris das Thymin, und sie schlossen daraus, daß die pflanzlichen Nucleinsäuren sich von den tierischen dadurch unterscheiden, daß die einen das Thynin, 
die anderen das Uracil in ihrem Moleküle enthielten. Eine eingehendere Nachforschung der Frage schien wünschenswert.

Die Hefenucleinsäure wurde in derselben Weise, wie es in der zweiten Mitteilung angegeben ist, dargestellt, die Spaltung mit $25 \%$ iger Schwefelsäure bei $175^{\circ} \mathrm{C}$. im Autoklaven ausgeführt Zur Darstellung und Trennung der Pyrimidinbasen war dasselbe Verfahren wie bei der Pankreasnucleinsäure benutzt. Es schied sich aber kein Thymin aus. Die Lösung wurde deshalb bald mit Picrinsäure behandelt und über Nacht stehen gelassen. Es bildete sich dabei ein Niederschlag. Aus der Mutterlauge schied sich beim Eindampfen bei vermindertem Druck ein zweiter Niederschlag aus. Die Picrate konnten leicht von der Picrinsäure mit Schwefelsäure, Toluol und Äther befreit werden. Der Überschuß der Schwefelsäure wurde mit Baryt entfernt und das Filtrat eingedampft. Beim Stehen schieden sich lange prismatische Nadeln aus, die, aus Wasser umkrystallisiert, die Zusammensetzung des basischen Cytosinsulfats besaßen. Die Substanz wurde im Toluolbad getrocknet.

$0,1365 \mathrm{~g}$ der Substanz gaben $33,5 \mathrm{ccm}$ Stickstoff bei $\mathrm{p}=768$ und $\mathrm{t}^{0}=19^{\circ} \mathrm{C}$.

$0,1470 \mathrm{~g}$ der Substanz gaben $0,0595 \mathrm{~g} \mathrm{BaSO}_{4}$.

Berechnet für $4\left(\mathrm{C}_{4} \mathrm{H}_{5} \mathrm{~N}_{3} \mathrm{O}\right) \cdot \mathrm{H}_{8} \mathrm{SO}_{4} \cdot 2 \mathrm{H}_{8} \mathrm{O}$ :

$\mathrm{N} \quad 29,06 \%$

S $5,53 \%$

Gefunden :

$29,08 \%$

$5,56 \%$

Ein Teil des Sulfats wurde in Lösung gebracht, mit Baryt von Schwefelsäure quantitativ befreit, und Filtrat bei vermindertem Druck eingedampft. Es schieden sich dabei Krystalle aus, die das Aussehen des Cytosins hatten und bei der Analyse die folgenden Zahlen gaben:

0,1865 g lufttrockener Substanzen verloren im Toluolbad getrocknet $0,345 \mathrm{~g}$.

Berechnet für $\mathrm{C}_{4} \mathrm{H}_{5} \mathrm{~N}_{3} \mathrm{O}+2 \mathrm{H}_{2} \mathrm{O}$ : Gefunden:

$$
\mathrm{H}_{2} \mathrm{O} \quad 24,49 \% \quad 23,46 \%
$$

Das Präparat schien also mit 2 Molekülen Wasser zu krystallisieren. Die zur Analyse angewandte Menge war aber zu klein, es mit Sicherheit zu schließen:

$0,1020 \mathrm{~g}$ der Substanz, im Toluolbad getrocknet, gaben $33,50 \mathrm{ccm}$ Stickstoff bei $p=767$ und $t^{0}=21,5$. 
8 P. A. Levene, Darstellung und Analyse einiger Nucleinsäuren.

$$
\begin{array}{cc}
\text { Berechnet für } \mathrm{C}_{4} \mathrm{H}_{5} \mathrm{~N}_{3} \mathrm{O}: & \text { Gefunden: } \\
\mathrm{N} 37,95 \% & 38,53 \%
\end{array}
$$

Ein zweiter Teil des Sulfats wurde in das Platinat übergeführt.

$0,1380 \mathrm{~g}$ der Substanz gaben $0,0425 \mathrm{~g}$ Pt.

Berechnet für $2\left(\mathrm{C}_{4} \mathrm{H}_{5} \mathrm{~N}_{3} \mathrm{O}\right) \mathrm{PtCl}_{4} \cdot 2 \mathrm{HGl}$ :

$\mathrm{Pt} \quad 30,84 \%$

Gefunden:

$30,79 \%$

Die Lösung der freien Base wie des Sulfats gaben mit Jodwismuthjodkaliumlösung einen ziegelroten Niederschlag. Die Mutterlauge des Cytosinpicrats wurde von der Picrinsäure und von der überschüssigen Schwefelsäure in üblicher Weise befreit und bei vermindertem Druck eingedampft, es schied sich dabei ein Niederschlag mit den Eigenschaften des Uracils aus:

$0,1425 \mathrm{~g}$ der Substanz gaben bei $\mathrm{p}=766$ und $\mathrm{t}^{\circ}=20,5^{\circ} \mathrm{C} .31,00 \mathrm{~cm}$ Stickstoff (über $50 \%$ Kalilauge).
Berechnet für $\mathrm{C}_{4} \mathrm{H}_{4} \mathrm{~N}_{2} \mathrm{O}_{2}$ : Gefunden:
N 25,05 25,53

Da man nicht mit Sicherheit schließen konnte, daß das Thymin nicht in der Mutterlauge vom Uracil zurückgeblieben war, so wurde sie mit Silber und Baryt behandelt. Es entstand dabei ein ganz kleiner Niederschlag, aus welchem kein Thymin zu gewinnen war.

Es waren etwa $150 \mathrm{~g}$ der Nucleinsäure verbraucht, und dabei entstanden:

$$
\begin{array}{ll}
\text { Uracil } & 5 \mathrm{~g} \\
\text { Cytosinpicrat } & 7
\end{array}
$$

Man scheint also zur Annahme berechtigt, daß die Hefenucleinsäure in ihrem Moleküle kein Thymin, aber außer Uracil auch Cytosin enthält.

\section{Literatur:}

Ascoli, Diese Zeitschrift, Bd. XXXI.

Kossel und Neumann, Ber. d. deutsch. ch. Ges., Bd. 27.

Kossel und Steudel, Diese Zeitschrift, Bd. XXXVII.

Levene, ibid. Bd. XXXVII. 